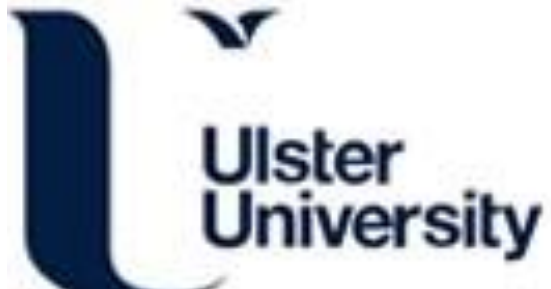

\section{Characterization of microwave absorption in carbon nanotubes using resonance aperture transmission method}

\author{
Malyuskin, O., Brunet, P., Mariotti, D., McGlynn, R., \& Maguire, P. (2020). Characterization of microwave \\ absorption in carbon nanotubes using resonance aperture transmission method. Journal of Applied Physics, \\ 128(4), 045109. [045109]. https://doi.org/10.1063/5.0002414
}

Link to publication record in Ulster University Research Portal

Published in:

Journal of Applied Physics

Publication Status:

Published online: 24/07/2020

DOI:

10.1063/5.0002414

\section{Document Version}

Publisher's PDF, also known as Version of record

\section{General rights}

Copyright for the publications made accessible via Ulster University's Research Portal is retained by the author(s) and / or other copyright owners and it is a condition of accessing these publications that users recognise and abide by the legal requirements associated with these rights.

\section{Take down policy}

The Research Portal is Ulster University's institutional repository that provides access to Ulster's research outputs. Every effort has been made to ensure that content in the Research Portal does not infringe any person's rights, or applicable UK laws. If you discover content in the Research Portal that you believe breaches copyright or violates any law, please contact pure-support@ulster.ac.uk. 


\section{Characterization of microwave absorption in carbon nanotubes using resonance aperture transmission method}

Cite as: J. Appl. Phys. 128, 045109 (2020); https://doi.org/10.1063/5.0002414

Submitted: 24 January 2020 . Accepted: 03 July 2020 . Published Online: 24 July 2020

O. Malyuskin (D), P. Brunet, D. Mariotti (D), R. McClynn (D), and P. Maguire (D)

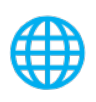

View Online

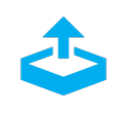

Export Citation

\section{ARTICLES YOU MAY BE INTERESTED IN}

Finite elements computational modeling of coupled elastic waveguides

Journal of Applied Physics 128, 045110 (2020); https://doi.org/10.1063/1.5127207

Phonon localization in single wall carbon nanotube: Combined effect of ${ }^{13} \mathrm{C}$ isotope and vacancies

Journal of Applied Physics 128, 045108 (2020); https://doi.org/10.1063/5.0011810

Acoustic radiation force and motion of a free cylinder in a viscous fluid with a boundary defined by a plane wave incident at an arbitrary angle

Journal of Applied Physics 128, 044902 (2020); https://doi.org/10.1063/5.0005866

\section{Lock-in Amplifiers up to $600 \mathrm{MHz}$}
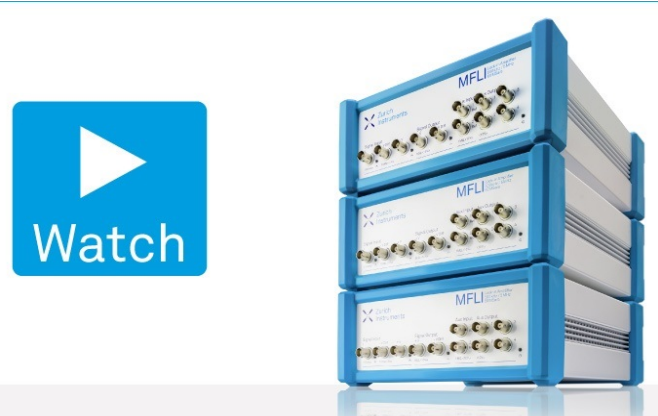


\title{
Characterization of microwave absorption in carbon nanotubes using resonance aperture transmission method
}

Cite as: J. Appl. Phys. 128, 045109 (2020); doi: 10.1063/5.0002414

Submitted: 24 January 2020 - Accepted: 3 July 2020 .

Published Online: 24 July 2020

O. Malyuskin, ${ }^{1, a)}$ (D) P. Brunet, ${ }^{2}$ D. Mariotti, ${ }^{2}$ (D) R. McGlynn, ${ }^{2}$ (D) and P. Maguire ${ }^{2}$ (D)

\author{
AFFILIATIONS \\ ${ }^{7}$ ECIT, Queen's University Belfast, Belfast BT3 9DT, United Kingdom \\ ${ }^{2}$ NIBEC, Ulster University, Newtownabbey BT37 OQB, United Kingdom
}

${ }^{a)}$ Author to whom correspondence should be addressed: $\circ$.malyuskin@qub.ac.uk

\begin{abstract}
A new method to characterize microwave electromagnetic (EM) absorption of a bulk carbon nanotube (CNT) material is proposed and experimentally evaluated in this paper. The method is based on the measurement of microwave transmission through a capacitive-resonator aperture (CRA) in a conductive screen loaded with a CNT sample under test. This method allows us to measure microwave permittivity and absorption of thin samples $(\sim 0.1 \mu \mathrm{m}-10 \mu \mathrm{m}$ thick) with linear dimensions much smaller than the wavelength of radiation in free space. This "minimal" sample requirement restricts the application of conventional microwave characterization methods such as free-space or waveguide permittivity characterization. It is demonstrated that the resonance E-field enhancement inside the CRA leads to strong EM interaction of the microwave Efield with the CNT sample under test, thus enabling high sensitivity and dynamic range $(\sim 5 \mathrm{~dB})$ of the measurement procedure. Another advantage of the proposed technique over conventional non-resonance characterization methods is that in the resonance transmission band, the CRA operation is reflection-less, which leads to a relatively simple qualitative algebraic de-embedding procedure of the material parameters based on the principle of energy conservation. The experimental microwave absorption data of the multiwall CNT samples are presented in the $\mathrm{S}$ frequency band $(2-4 \mathrm{GHz})$, demonstrating microwave absorption properties of the multiwall CNT ribbons.
\end{abstract}

Published under license by AIP Publishing. https://doi.org/10.1063/5.0002414

\section{INTRODUCTION}

The development of advanced composite and nano-materials requires a range of measurement techniques to characterize the electrical, electronic, mechanical, and thermal properties of the material. One important class of materials is represented by thin films and self-standing nano-materials with sample thickness varying from mono-atomic layers to few micrometers with relatively large, macroscopic area dimensions. An example of such a material is carbon nanotube (CNT) ribbons that exhibit very unique mechanical, electric, and electronic properties.

CNTs have a wide range of unique applications in nano- and microelectronics, radiation detection, chemical and bio-sensing, composite materials, etc. ${ }^{2,3}$ In this letter, a new method to characterize electromagnetic (EM) properties of a bulk CNT material in the microwave range is proposed. The method is based on the reflection-less resonance transmission of microwave radiation through the capacitive-resonator apertures (CRAs) with and without a CNT sample. From the measurement data, real and imaginary parts of the CNT complex permittivity can be extracted using differential material parameters de-embedding. ${ }^{4,5}$ Permittivity of the pure CNT can be further employed to design EM absorbers and EM interference shielding based on the composite materials formed by the CNT material inclusions inside a host medium, e.g., epoxy or resins. ${ }^{6,7}$ These composite materials can be promising for advanced aerospace applications ${ }^{3,6-9}$ and future wireless communications, especially, for the design of the intelligent EM-controlled metasurfaces, ${ }^{8}$ due to mechanical and chemical stability and very low weight density of the CNTs.

The existing EM material characterization methods ${ }^{10-14}$ can be divided into several groups including free-space, waveguide, resonating cavity, 3D resonator, transmission line, open coaxial, evanescent probe, and a parallel-plate capacitor method. The discussion of the application range, advantages, and drawbacks of these methods can be found elsewhere. ${ }^{10-14}$ As a rule, most of the above-mentioned 
methods require sufficiently large CNT samples covering the area of at least $0.25 \lambda \times 0.5 \lambda$, where $\lambda$ is a wavelength of radiation in free space. Also, the measurement of thin samples (thickness of few micrometers or less) represents a significant challenge for free-space or waveguide methods in the microwave range ( $\lambda$ is in the range of $0.1 \mathrm{~cm}-30 \mathrm{~cm}$ ) due to low sensitivity and numerical errors in permittivity de-embedding algorithm in the thin sample permittivity reconstruction scenario. ${ }^{1}$

Resonance CRA transmission method discussed here offers a number of distinctive advantages: high sensitivity and dynamic range of the method are due to EM field enhancement inside the CRA leading to strong EM interaction between the testing microwave and even very thin samples. The electrically small size of the CRA allows us to use "minimal" thin CNT samples with characteristic 2D dimensions significantly smaller than the radiation wavelength.

\section{GEOMETRY OF THE MEASUREMENT SETUP}

The measurement setup geometry is shown in Fig. 1. The setup consists of a pair of dipole antennas and a CRA in the conductive screen loaded with a thin CNT material sample. The transmit (TX) and receive (RX) microwave signals are generated and received by the vector network analyzer (VNA).

This material characterization method is based on differential measurements, when the transmission data through the loaded and unloaded aperture are required along with the measurement of the free-space transmission link between the RX and TX antennas, in order to de-embed antenna characteristics and free-space propagation link properties from the overall measurement system transmission.

\section{ELECTROMAGNETIC MODEL OF THE CRA MICROWAVE TRANSMISSION}

Microwave transmission through the CRA aperture can be described $^{10,12,15}$ by the system of Eqs. (1) and (2),

$$
\boldsymbol{E}_{t F S}(\boldsymbol{r})=\hat{T}\left(\varepsilon_{0}\right) \cdot \boldsymbol{E}_{i}\left(\boldsymbol{r}_{\boldsymbol{A}}\right)
$$

$$
\boldsymbol{E}_{t C N T}(\boldsymbol{r})=\hat{T}\left(\varepsilon_{C N T}\right) \cdot \boldsymbol{E}_{i}\left(\boldsymbol{r}_{\boldsymbol{A}}\right),
$$

where $\boldsymbol{E}_{t F S}$ is a TX microwave $E$-field through the unloaded (in the air) CRA, $\boldsymbol{E}_{t C N T}(\boldsymbol{r})$ is a TX $E$-field through the CRA loaded with a CNT sample, $\boldsymbol{r}$ is a radius-vector of observation point in the $\mathrm{z}>0$ half-space, and $\boldsymbol{r}_{\boldsymbol{A}}$ is a radius-vector of observation point across the CRA aperture, in the plane $z=0$, Fig. $1(b) . \hat{T}$ is a second-rank $(3 \times 3)$ tensor operator describing microwave TX properties of the CRA. $\boldsymbol{E}_{i}$ is the incident EM field generated by a TX antenna with the center located at $\mathrm{z}=-\mathrm{z}_{\mathrm{d}}$. To derive the transmission equations in terms of the measurable RX power, the TX antenna is characterized by its radiation gain $\boldsymbol{G}_{T X}\left(\boldsymbol{r}, \boldsymbol{r}_{T X}\right)$, where $\boldsymbol{r}_{T X}$ is a radius-vector of the TX antenna center. The dipole RX antenna can be characterized by its effective vector length, $\boldsymbol{l}_{R X}$, relating RX voltage across the RX antenna terminal and the E-field acting on the antenna. Assuming that the input impedances of the $\mathrm{RX}$ and TX antennas are the same $(50 \Omega)$, it is straightforward to derive the transmission equation in terms of the TX and RX power and EM transmission tensor $\hat{T}$ of the CRA,

$$
P_{R X}=\left(\hat{T} \cdot \boldsymbol{G}_{T X} \cdot \boldsymbol{l}_{R X}\right)^{2} P_{T X}
$$

The differential measurement procedure is based on the normalization of the transmitted power through the CNT-loaded CRA, $P_{R X, C N T}$, by the transmitted power through the unloaded CRA, $P_{R X, F S}$ for the same input power $P_{T X}$,

$$
P_{R X, C N T} / P_{R X, F S}=\left[T\left(\varepsilon_{C N T}\right) / T\left(\varepsilon_{0}\right)\right]^{2},
$$

where $T\left(\varepsilon_{C N T}\right)$ and $T\left(\varepsilon_{0}\right)$ are the EM transfer functions of the loaded and unloaded CRA for the vertically y-polarized $E$-field. Differential measurement method allows us to eliminate RX and TX antenna characteristics from the CNT permittivity de-embedding procedure which employs normalized transmitted power function (4).

The microwave transmission function of the CRA appeared in (1), (2) is an integral-differential tensor operator, whose form

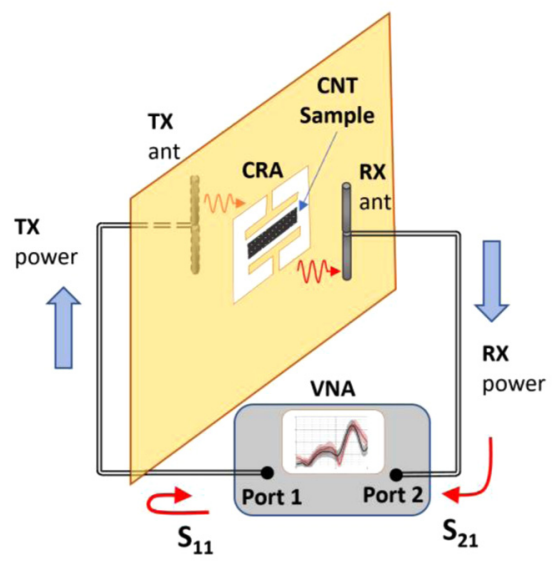

(a)

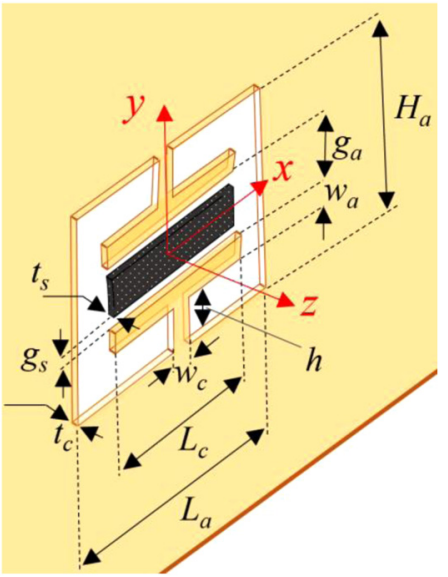

(b)
FIG. 1. (a) Measurement setup geometry and (b) detailed view of the CNT-loaded aperture with dimensions. The measurement setup consists of a CRA, a pair of transmit (TX) and receive (RX) dipole antennas, and a vector network analyzer (VNA) to measure the microwave S-parameters. 
can be established using the EM field potential equations. For the monochromatic, $\exp (-i \omega t)$, time dependence the EM field transmitted through the material-loaded aperture can be written in the form ${ }^{16,17}$

$$
\boldsymbol{E}_{t}(\boldsymbol{r})=\frac{i}{\omega \mu \varepsilon_{n}}\left(\nabla \nabla+k_{n}^{2}\right) \cdot \boldsymbol{A}-\frac{1}{\varepsilon_{n}} \nabla \times \boldsymbol{F} .
$$

In (5), $\mu$ is a magnetic permeability of free space, index $n$ defines the material, $n=\mathrm{CNT}$ or $n=$ free space or air, $k_{n}=\sqrt{\varepsilon_{n}} \omega / c$, $c$ is a speed of light in vacuum, and $\mathbf{A}$ and $\mathbf{F}$ are the EM vectorpotentials, $^{17}$

$$
\begin{gathered}
\boldsymbol{A}(\boldsymbol{r})=\mu \int_{\text {Apert. }} G_{n}\left(\boldsymbol{r}, \boldsymbol{r}^{\prime}\right) \boldsymbol{J}\left(\boldsymbol{r}^{\prime}\right) d \boldsymbol{r}^{\prime}, \\
\boldsymbol{F}(\boldsymbol{r})=\varepsilon_{n} \int_{\text {Apert. }} G_{n}\left(\boldsymbol{r}, \boldsymbol{r}^{\prime}\right) \boldsymbol{J}_{m}\left(\boldsymbol{r}^{\prime}\right) d \boldsymbol{r}^{\prime} .
\end{gathered}
$$

In (6), the integration is carried out over the volume occupied by the CNT material, Fig. 1(b), and involves the electric polarization current density $J$,

$$
\boldsymbol{J}(\boldsymbol{r})=-i \omega \varepsilon_{0}\left(\varepsilon_{r C N T}-1\right) \boldsymbol{E}_{a}(\boldsymbol{r}),
$$

where $\varepsilon_{r C N T}=\varepsilon_{C N T}^{\prime}+i \varepsilon_{C N T}^{\prime \prime}$ is a complex-valued relative permittivity of CNT and $\boldsymbol{E}_{a}(\boldsymbol{r})$ is an E-field inside the CNT volume. In (7), the area integration is carried out inside the CRA area
$-L_{a} / 2<x<L_{a} / 2,-H_{a} / 2<y<H_{a} / 2$, free from the CNT or conductor, at the plane $\mathrm{z}=t_{c}$, and involves magnetic current density $\boldsymbol{J}_{m}$,

$$
\boldsymbol{J}_{m}(\boldsymbol{r})=-\boldsymbol{z}_{0} \times \boldsymbol{E}_{a}(\boldsymbol{r}) .
$$
relation,

Finally, scalar Green's function in (6), (7) is given by a

$$
G\left(\boldsymbol{r}, \boldsymbol{r}^{\prime}\right)=\exp \left(i k_{n}\left|\boldsymbol{r}-\boldsymbol{r}^{\prime}\right|\right) / 4 \pi\left|\boldsymbol{r}-\boldsymbol{r}^{\prime}\right| .
$$

The explicit form of the transfer function $T$ in (1), (2) can be established by using permittivity $\varepsilon_{C N T}$ in (5)-(10) and calculating the E-field $\boldsymbol{E}_{a}\left(\boldsymbol{r}_{\boldsymbol{A}}\right)$ inside the CRA from the boundary conditions and Eqs. (6) and (7). It should also be noted that the real $\varepsilon_{C N T}^{\prime \prime}$ and imaginary $\varepsilon_{C N T}^{\prime \prime}$ parts of the CNT permittivity $\varepsilon_{C N T}$ appear in the transmission Eq. (4) in a coupled form, thus permittivity de-embedding requires the inverse problem solution of (1)-(10) based on the full-wave numerical computation. Below, an analytical solution based on the energy conservation principle is proposed which can also serve as an initial approximation in the full-wave numerical solution to (1)-(10).

\section{ANALYTICAL SOLUTION FOR THE CNT PERMITTIVITY DE-EMBEDDING}

To obtain an approximate analytical solution to the permittivity of the CNT sample under test, unloaded and loaded CRAs can be represented as equivalent circuits, ${ }^{18}$ Fig. 2.
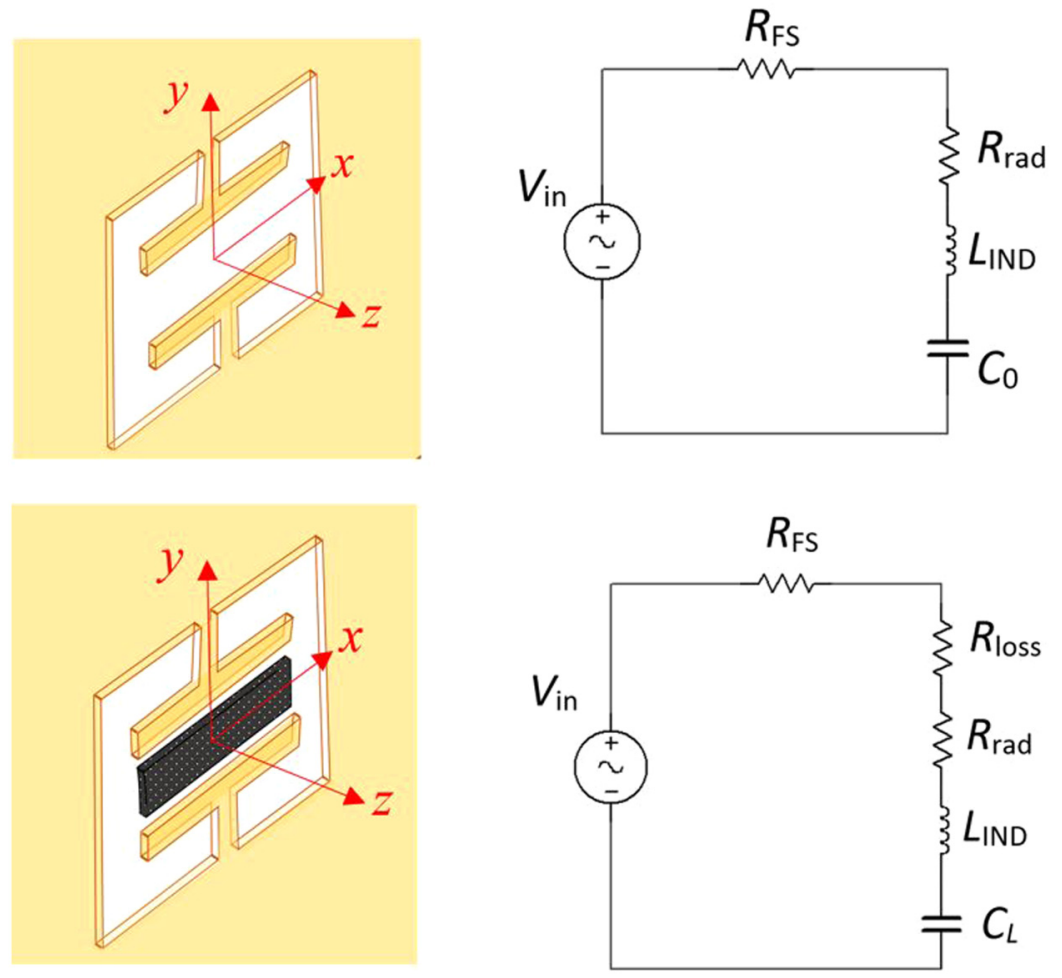

FIG. 2. Equivalent lump circuit model of an unloaded (top) and CNT-loaded (bottom) CRA. 
TABLE I. Dimensions of the CRA (mm).

\begin{tabular}{lllllll}
\hline \hline No. CRA & $\mathrm{L}_{\mathrm{a}}$ & $\mathrm{H}_{\mathrm{a}}$ & $\mathrm{L}_{\mathrm{c}}$ & $\mathrm{g}_{\mathrm{a}}$ & $\mathrm{w}_{\mathrm{a}}$ & $\left\langle\mathrm{g}_{\mathrm{s}}>\right.$ \\
\hline 1 & 30 & 22 & 28 & 6 & 2 & 0.2 \\
2 & 26 & 18 & 24 & 6 & 2 & 0.3 \\
3 & 22 & 16 & 20 & 6 & 2 & 0.5 \\
4 & 20 & 15 & 18 & 6 & 2 & 0.8 \\
\hline \hline
\end{tabular}

In these circuits, source $V_{\text {in }}$ models TX antenna, $R_{\mathrm{FS}}$ stands for the free-space characteristic impedance, $R_{\text {rad }}$ is CRA radiation resistance, $L_{\mathrm{IND}}$ is a CRA equivalent inductance, and $C_{0}$ and $C_{\mathrm{L}}$ are unloaded and loaded equivalent capacitance. $R_{\text {loss }}$ models the microwave loss due to conductivity (imaginary part of the permittivity) of the CNT sample, while loaded capacitance $C_{\mathrm{L}}$ is a real-valued function of CRA geometry and real part of the CNT permittivity. Since the CNT material studied in this work is a lossy non-magnetic or weakly magnetic dielectric, the equivalent inductance of the loaded and unloaded aperture is approximately the same. The exact form of the equivalent circuit lump parameters can be established using surface currents and charge density ${ }^{16,17}$ across the CRA; however, this is not required for the analytical model described below.

Using resonance circuit models in Fig. 2, it can be seen that CRA loading with a CNT sample results in the microwave transmission resonance frequency shift,

$$
f_{0}^{2} / f_{L}^{2}=C_{L} / C_{0}
$$

where $f_{0}$ and $f_{L}$ are the resonance frequencies of the unloaded and loaded CRA, respectively. Due to the high-quality resonance of the CRAs used in this work (measured Q-factor typically exceeds 10), resonance frequency pulling, ${ }^{19} f_{L}^{\text {loss }}$,

$$
f_{L}^{\text {loss }}=f_{0} \sqrt{1-1 / 4 Q^{2}}
$$

due to microwave loss caused by the CNT absorption is small (less than 5\%), and, therefore, the real part of CNT permittivity plays a dominant role in the CRA transmission resonance frequency change. To derive analytical expressions for the resonance frequency shift as a function of the CNT permittivity real part, CNT-loaded and unloaded capacitance of a CRA can be evaluated using a quasi-static approximation (please refer to Appendix A in the supplementary material for details),

$$
\left(\varepsilon_{r C N T}^{\prime}+A_{0}\right) /\left(1+A_{0}\right)=f_{0}^{2} / f_{L}^{2},
$$

where the constant $A_{0}=\pi g_{a} /\left(w_{a} \ln \left(1+g_{a} / w_{a}\right)\right)$ depends on the CRA gap to width of the plate's ratio. Formula (12) is obtained in the assumption that the CNT material fills uniformly the gap space $-L_{c} / 2 \leq x \leq L_{c} / 2, \quad-\frac{g_{a}}{2} \leq y \leq \frac{g_{a}}{2}, 0 \leq z \leq t_{c}$ between the CRA plates 1 and 2, Fig. S1, Appendix A in the supplementary material.

The imaginary part of the CNT sample permittivity can be found from the power conservation principle, which results in the Eq. (13), the detailed derivation is provided in Appendix B in the supplementary material,

$$
\varepsilon_{r C N T}^{\prime \prime}=\varepsilon_{r C N T}^{\prime}\left[W_{1} P_{R X}\left(f_{0}\right)-W_{2} P_{R X}\left(f_{L}\right)\right] .
$$

In Eq. (13),

$$
W_{1}=\frac{D\left(f_{L}\right)}{D^{2}\left(f_{0}\right)} \frac{T\left(f_{L}\right)}{T\left(f_{0}\right)} \frac{1+Q_{L}}{1+Q_{0}} R\left(f_{0}\right), \quad W_{2}=\frac{R\left(f_{L}\right)}{D\left(f_{L}\right)} .
$$

Equations (13) and (14) involve measurable parameters: $D\left(f_{0}\right)$ and $D\left(f_{L}\right)$ are the propagation path loss factors between the TX antenna and the CRA plane at resonance frequencies $f_{0}$ and $f_{L}$, respectively, coefficients $T$ describe TX antenna matching, $T(f)=\left|1-S_{11}(f)\right|$, coefficients $R$ characterize matching of the RX antenna at frequency $f, R(f)=\left|1-S_{22}(f)\right|^{-1}$, Q-factors of the loaded $\left(Q_{L}\right)$ and unloaded $\left(Q_{0}\right)$ CRAs can be calculated from the CRA transmission power characteristics in standard form as a ratio of the resonance frequency to the $3 \mathrm{~dB}$ bandwidth, $Q=f_{\text {res }} / \Delta f, P_{R X}(f)$ is the RX power measured by the RX antenna at frequency $f$, normalized to the input power at TX antenna. Equations (12) and (13) allow us to de-embed the real $\varepsilon_{r C N T}^{\prime}$ and imaginary $\varepsilon_{r C N T}^{\prime \prime}$ parts of the CNT relative permittivity $\varepsilon_{r C N T}$ based on algebraic equations.
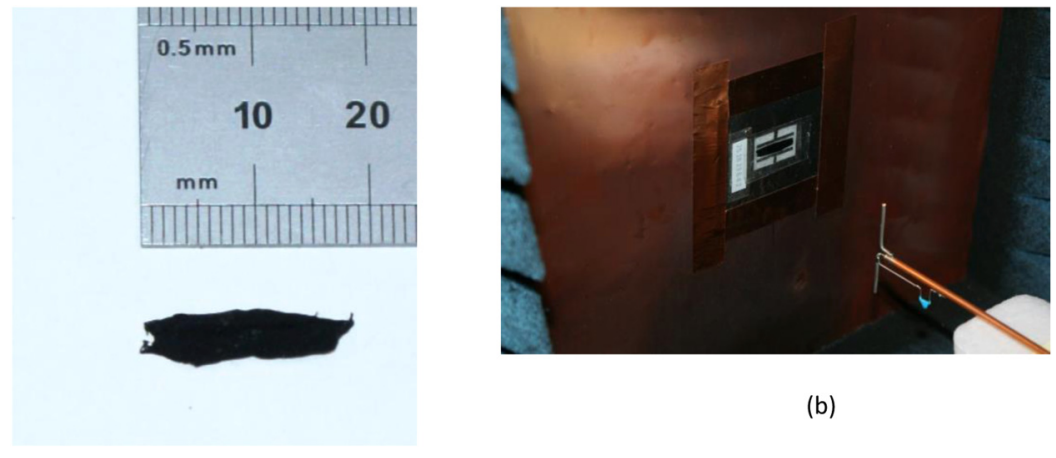

(b)
FIG. 3. (a) Example of a CNT sample and (b) CNT-loaded CRA and TX dipole antenna inside the anechoic environment. 
TABLE II. Measured resonance frequency, Q-factors, RX power, and de-embedded permittivity of CNT.

\begin{tabular}{lcccccccc}
\hline \hline No. & $f_{0}$ & $f_{L}$ & & & $\begin{array}{c}P_{R X} \\
\left(f_{0}\right)\end{array}$ & $\begin{array}{c}P_{R X} \\
\left(f_{L}\right)\end{array}$ & Re & $\operatorname{Im}$ \\
CRA & $(\mathrm{GHz})$ & $(\mathrm{GHz})$ & $Q_{0}$ & $Q_{L}$ & $(\mathrm{~dB})$ & $(\mathrm{dB})$ & $\varepsilon_{C N T}$ & $\varepsilon_{C N T}$ \\
\hline 1 & 1.97 & 1.71 & 9.34 & 5.89 & -25.25 & -37.02 & 3.55 & 3.97 \\
2 & 2.48 & 2.175 & 13.41 & 14.51 & -32.71 & -37.82 & 3.34 & 2.09 \\
3 & 3.04 & 2.66 & 13.20 & 12.98 & -30.90 & -33.76 & 3.35 & 2.69 \\
4 & 3.27 & 2.94 & 16.86 & 13.386 & -32.82 & -33.10 & 2.85 & 1.86 \\
\hline \hline
\end{tabular}

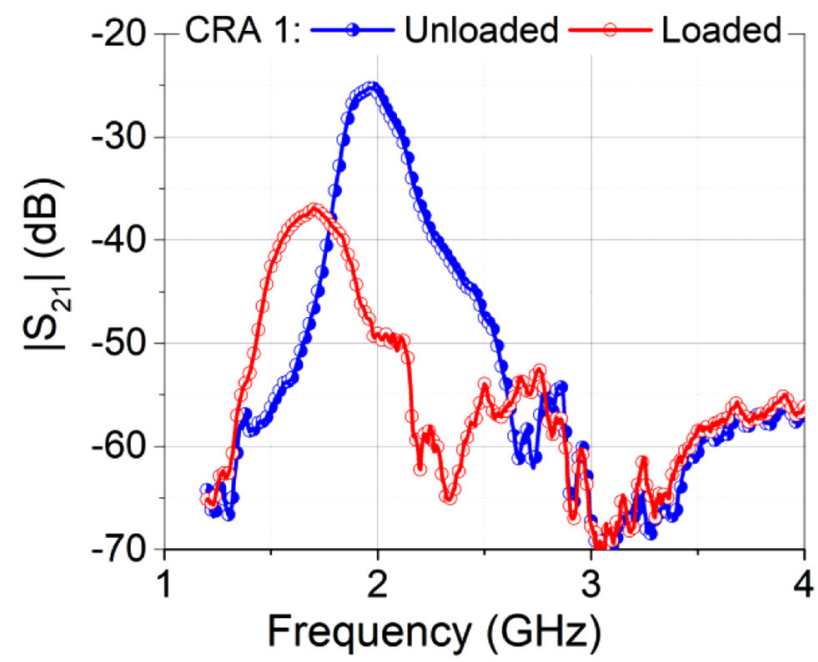

(a)

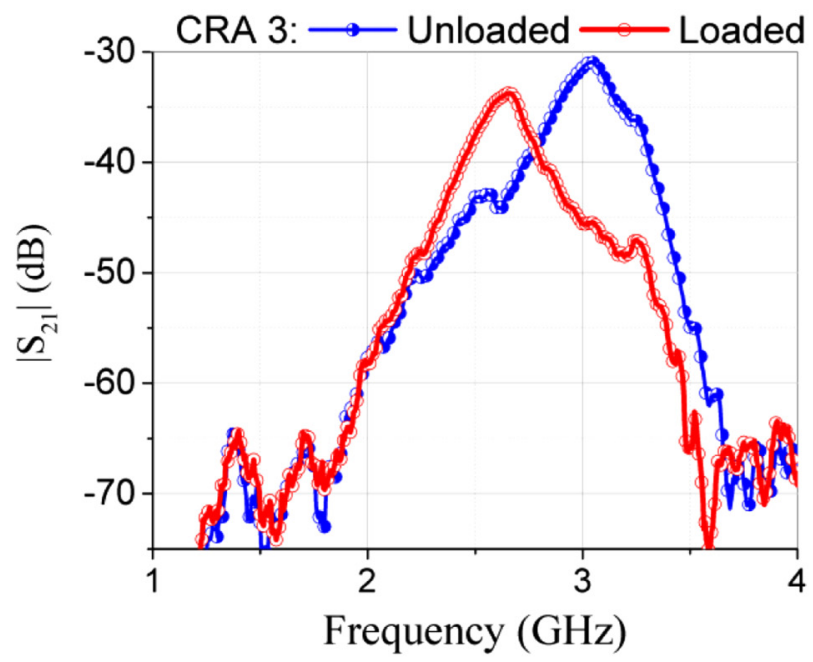

(c)

\section{EXPERIMENTAL RESULTS}

\section{A. Sample preparation}

CNTs were made in an axial tubular high temperature furnace at $1290^{\circ} \mathrm{C}$ known as a floating catalyst chemical vapor deposition (FC-CVD) in background hydrogen $(1350 \mathrm{sccm})$. The precursors used for the CNT production are ferrocene for the catalyst's nanoparticles, thiophene to supply sulfur as a growth limiter of the CNT diameter, and methane as a carbon precursor. Both ferrocene and thiophene are introduced via bubblers with hydrogen flow at $130 \mathrm{sccm}$ and $90 \mathrm{sccm}$, respectively. The bubbler with thiophene

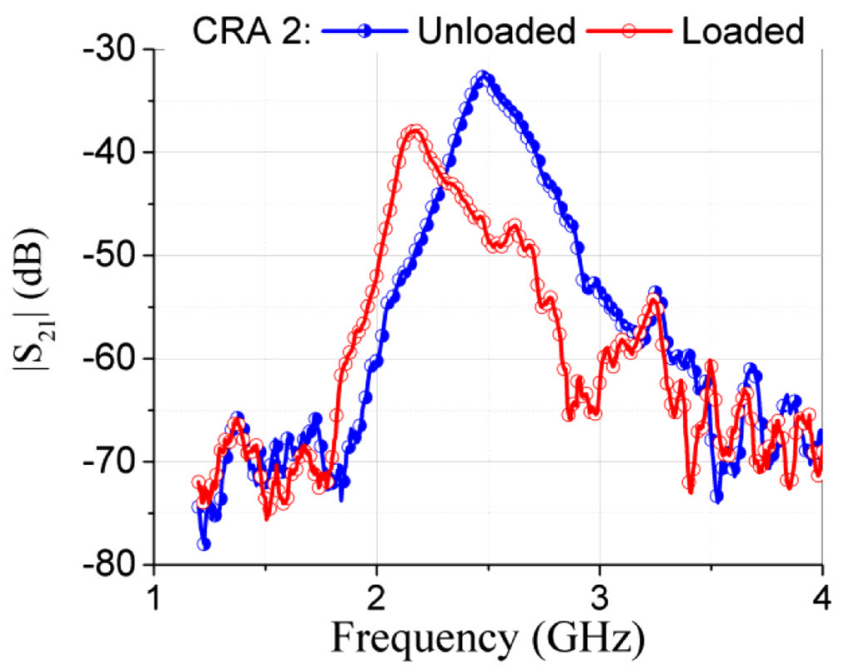

(b)

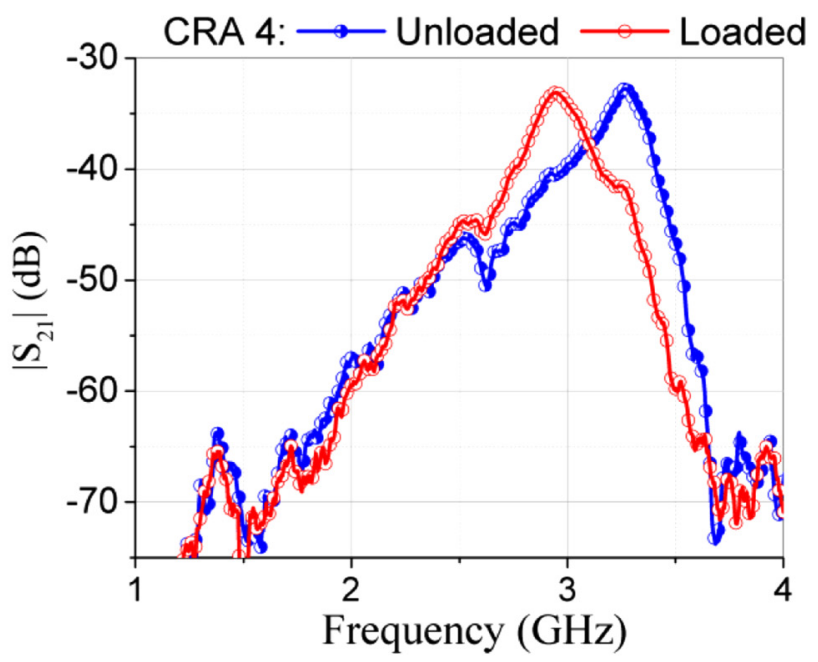

(d)

FIG. 4. (a)-(d) Measured transmission power functions $\left|S_{21}\right|$ of four fabricated CRAs, unloaded and loaded with a CNT sample. 
was kept at $25^{\circ} \mathrm{C}$. The decomposition of the ferrocene precursor leads to nucleation and growth of iron nanoparticles with the incorporation of sulfur, which has been shown to play an important role in these mechanisms. ${ }^{20,21}$ Methane flow was set at $160 \mathrm{sccm}$. During the synthesis process, an aerogel forms at the exit of the chamber and this is collected and pulled out from the furnace by the help of a rod. This process allows the formation of macroscopic assemblies of multiwall CNTs that can be adapted in different morphologies. In this case, CNTs have been mechanically compacted and cut in length to produce CNT ribbons of a few centimeters and a thickness with the range of $1-4 \mu \mathrm{m}$.

The CRAs have been fabricated using a thin aluminum foil of approximately $10 \mu \mathrm{m}$ thickness. The CNT samples were positioned on the Rohacell $5 \mathrm{~mm}$-thick substrate IG51 (permittivity 1.071, $\tan \delta=0.0031$ ) and affixed with the Scotch tape (permittivity 1.9, $\tan \delta=0.006$ ). Four CRAs were fabricated with the dimensions summarized in Table I. In all CRAs, $w_{s}=w_{a},\left\langle\mathrm{~g}_{\mathrm{s}}\right\rangle$ is an average value of gap $g_{s}$.

\section{B. Microwave measurement data and analysis}

Two sets of dipole antennas were used to provide optimal matching in the bands $1.7-2.5 \mathrm{GHz}$ and $2.4-3.2 \mathrm{GHz}$. The return loss $\left|S_{11}\right|$ characteristics of the antennas in measurement setup are provided in Appendix $\mathrm{C}$ in the supplementary material. This choice of the frequency range is motivated by the practical interest in the WiFi band CNT characteristics and ease of the measurements at a lower frequency range. However, the design can be straightforwardly scaled for a higher frequency range.

The CRA transmission characteristics $\left|S_{21}\right|$ are presented in Fig. 4. Table II summarizes the measured parameters: resonance frequency, $Q$-factors, RX power, and de-embedded relative CNT permittivity using an approximate model (13) and (14). The dipole antennas are fed with $-10 \mathrm{dBm}$ power (at the VNA output).

The $\left|S_{21}\right|$ transmission characteristics presented in Fig. 4 demonstrate the very high dynamic range and sensitivity of the CRA to the CNT sample loading, the resonance frequency shift in all cases is around $11 \%-14 \%$, RX power variation between unloaded and loaded CRA $\left|S_{21}\right|$ is approximately $2-10 \mathrm{~dB}$ which is due to field enhancement inside the CRA (please see more detail in Appendix D in the supplementary material). The permittivity data calculated in Table II are consistent with the previous measurements carried out by other authors, ${ }^{22-26}$ it is believed that the major absorption mechanism in the studied CNT ribbons is due to CNT conductivity losses. ${ }^{26}$

It should be noted that additional measurements not presented here for the brevity sake demonstrate the CRA transmission sensitivity to the gap $\mathrm{g}_{\mathrm{s}}$ between the CNT sample and the CRA plates. This gap leads to the lower values of permittivity as can be understood from the effective medium approximation; ${ }^{27-29}$ therefore, the gap $g_{s}$ should be as minimal as possible for accurate permittivity characterization. The major drawback of the proposed method in its present form is a requirement for broadband dipole antennas matched in the wide frequency band. This drawback can be eliminated by using printed resonators fed by broadband printed strip lines and incorporating a printed array of the CRA resonators on a single printed circuit board.

\section{CONCLUSIONS}

A new method to characterize permittivity of the CNT samples using resonance, reflection-less CRA transmission is proposed. Initial experimental data demonstrate high sensitivity and dynamic range of the proposed method, which is practically important for the EM characterization of thin "minimal" samples of the CNT material. The approximate analytical procedure of the permittivity de-embedding from the measurement data is proposed, reconstructed permittivity values are consistent with the previous measurements. This method can be easily scaled in the frequency range (from microwave to submillimetre wavelengths), thus providing an accurate EM characterization tool in addition to the existing methods.

\section{SUPPLEMENTARY MATERIAL}

The supplementary material includes calculation of the CRA capacitance, derivation of the power balance equation, the measured S-parameters of the TX dipole antennas, and the simulation results demonstrating E-field enhancement inside the CRA.

\section{DATA AVAILABILITY}

The data that support the findings of this study are available within the article and its supplementary material.

\section{REFERENCES}

${ }^{1}$ J. N. Wang, X. G. Luo, T. Wu et al., "High-strength carbon nanotube fibre-like ribbon with high ductility and high electrical conductivity," Nat. Commun. 5, 3848 (2014).

${ }^{2}$ Carbon Nanotubes: Science and Applications, edited by M. Meyyappan (CRC Press, 2005).

${ }^{3}$ Advanced Nanomaterials for Aerospace Applications, edited by C. Cabrera and F. Miranda (Pan Stanford Publishing, 2014).

${ }^{4}$ K. Y. You, F. B. Esa, and Z. Abbas, "Macroscopic characterization of materials using microwave measurement methods-A survey," in PIERS - FALL, 194-204 (IEEE, 2017).

${ }^{\mathbf{5}} \mathrm{F}$. Costa, M. Borgese, M. Degiorgi, and A. Monorchio, "Electromagnetic characterisation of materials by using transmission/reflection (T/R) devices," Electronics 6, 95 (2017).

${ }^{6} \mathrm{H}$. Zhang, G. Zeng, Y. Ge, T. Chen, and L. Hu, "Electromagnetic characteristic and microwave absorption properties of carbon nanotubes/epoxy composites in the frequency range from 2 to $6 \mathrm{GHz}$," J. Appl. Phys. 105(5), 054314 (2009).

${ }^{7}$ X. Xia, Y. Wang, Z. Zhong, and G. J. Weng, "A theory of electrical conductivity, dielectric constant, and electromagnetic interference shielding for lightweight graphene composite foams,” J. Appl. Phys. 120, 085102 (2016).

${ }^{8} \mathrm{~L}$. Wang and Z.-M. Dang, "Carbon nanotube composites with high dielectric constant at low percolation threshold," Appl. Phys. Lett. 87, 042903 (2005).

${ }^{9} \mathrm{~A}$. Li, Z. Luo, H. Wakatsuchi et al., "Nonlinear, active, and tunable metasurfaces for advanced electromagnetics applications," IEEE Access 5, 27439-27452 (2017).

${ }^{10} \mathrm{~F}$. Gonçalves, A. Pinto, R. Mesquita et al., "Free-space materials characterization by reflection and transmission measurements using frequency by-frequency and multi-frequency algorithms," Electronics 7, 260 (2018).

${ }^{11}$ Y. Gao, M. T. Ghasr, M. Nacy, and R. Zoughi, "Towards accurate and wideband in vivo measurement of skin dielectric properties," IEEE Trans. Instrum. Meas. 68(2), 512-524 (2019).

12J. Baker-Jarvis, M. D. Janezic, and D. C. Degroot, "High-frequency dielectric measurements: Part 24 in a series of tutorials on instrumentation and measurement," IEEE Instrum. Meas. Mag. 13(2), 24-31 (2010). 
${ }^{13}$ L. Liu et al., "Microwave dielectric properties of carbon nanotube composites," in Carbon Nanotubes (Intech Open, 2010).

${ }^{14}$ L. Liu, L. Kong, W.-Y. Yin, and S. Matitsine, "Characterization of single- and multi-walled carbon nanotube composites for electromagnetic shielding and tunable applications," IEEE Trans. Electromagn. Compat. 53, 943-949 (2011).

${ }^{15} \mathrm{~J}$. Sheen, "Comparisons of microwave dielectric property measurements by transmission/reflection techniques and resonance techniques," Meas. Sci. Technol. 20, 042001 (2009).

${ }^{16} \mathrm{C}$. L. Gardner and G. I. Costache, "The penetration of EM waves through loaded apertures," IEEE Trans. Electromagn. Compat. 37(3), 358-366 (1995).

${ }^{17}$ J. A. Kong, Electromagnetic Wave Theory (Wiley-Blackwell, 2000).

${ }^{18} \mathrm{~A}$. W. Love, "Equivalent circuit for aperture antennas," Electron. Lett. 23(13), 708-710 (1987).

${ }^{19}$ Dielectric Resonators, edited by D. Kajfez and P. Guillon (Artech House, 1986).

${ }^{20} \mathrm{C}$. Hoecker et al., "The dependence of CNT aerogel synthesis on sulfur-driven catalyst nucleation processes and a critical catalyst particle mass concentration," Sci. Rep. 7(1), 14519 (2017).

${ }^{21} \mathrm{M}$. S. Motta et al. "The role of sulphur in the synthesis of carbon nanotubes by chemical vapour deposition at high temperatures," J. Nanosci. Nanotechnol. 8, 2442-2449 (2008).
${ }^{22}$ D. Micheli, R. Pastore, C. Apollo et al., "Broadband electromagnetic absorbers using carbon nanostructure-based composites," IEEE Trans. Microwave Theory Tech. 59, 2633-2646 (2011).

${ }^{23}$ P. Savi, M. Giorcelli, and S. Quaranta, "Multi-walled carbon nanotubes composites for microwave absorbing applications," Appl. Sci. 9, 851 (2019).

${ }^{24} \mathrm{Z}$. Wang and G. Zhao, "Microwave absorption properties of carbon nanotubes-epoxy composites in a frequency range of $2-20 \mathrm{GHz}$," Open J. Compos. Mater. 03(2), 17-23 (2013).

${ }^{25}$ A. Katsounaros, K. Z. Rajab, Y. Hao et al., "Microwave characterization of vertically aligned multiwalled carbon nanotube arrays," Appl. Phys. Lett. 98, 203105 (2011).

${ }^{26} \mathrm{M}$. Green and X. Chen, "Recent progress of nanomaterials for microwave absorption," J. Materiomics 5(4), 503-541 (2019).

${ }^{27} \mathrm{~F}$. Hu, J. Song, and T. Kamgaing, "Modeling of multilayered media using effective medium theory," in 19th Meeting on Electrical Performance of Electronic Packaging and Systems (IEEE, 2010), 225-228.

${ }^{28}$ R. Esquivel-Sirvent and G. C. Schatz, "Mixing rules and the Casimir force between composite systems," Phys. Rev. A. 83, 042512 (2011).

${ }^{29} \mathrm{~F}$. R. Zypman, "Mathematical expression for the capacitance of coplanar strips,” J. Electrost. 101, 103371 (2019). 